\title{
Numerical simulation research on permeability coefficient inversion based on the data of mine water inrush
}

\author{
Baofeng Zhao ${ }^{1,}$, Lianjing Ma 2, 3, b \\ ${ }^{1}$ Xi'an Research Institute, China Coal Technology and Engineering Group Corp., Xi'an 710077, \\ China \\ ${ }^{2}$ Chang'an University School of environmental science and engineering, Xi'an 710054, China \\ ${ }^{3}$ Key Laboratory of Subsurface Hydrology and Ecological Effects in Arid Region (Chang'an \\ University ), Ministry of Education, Xi'an 710054, China \\ a sunman1220@163.com, b860909mlj@163.com
}

Keywords: water inrush; permeability coefficient; numerical simulation; aquifer

Abstract. In order to find out the hydrogeological condition of aquifer and gain the permeability coefficient, the permeability coefficient was inverted based on the data of mine water inrush. The observation data of water flow and long-time observation hole's water level was be used during the process of water inrush, and Visual Modflow was used to research the permeability coefficient in the study area. The result shows that Zhiluo formation aquifer's permeability coefficient is $0.5 \sim 4.1 \mathrm{~m} / \mathrm{d}$, the water storage rate is $2 \times 10^{-6} \sim 7 \times 10^{-5}$. Through the analysis of permeability coefficient gained by water inrush data and previous hydrogeological work, the permeability coefficient of this research according to the actual situation, and can be used as the basis of mine water disaster prevention and control work.

\section{Introduction}

The permeability coefficient of aquifer is a parameter of rock permeability, it reflects the hydrogeological characteristics of aquifer, and it is an important parameter for groundwater resource evaluation and water disaster prevention and control in coal mine ${ }^{[1]}$. The methods to obtain the permeability coefficient include pumping test, injection test, seepage test and dewatering test. Pumping test is the most widely and common method to obtain hydrogeological parameters based on drilling the borehole on the ground, pumping groundwater with the borehole and observing the water level ${ }^{[2]}$. The principle of dewatering test is similar with pumping test: though drilling the borehole under the ground, observing the water flow and water level, the permeability coefficient is obtained. Dewatering test is suitable for the complicated hydrogeological conditions or the mining area without the condition of drilling the borehole on the ground.

The pumping test and dewatering test are the main way to obtain the permeability coefficient, but they need drilling the borehole specially. This paper tries to adopt the numerical simulation to determine the permeability coefficient based on observation data of water flow and water level during the process of water inrush. This method avoids the input of quantities and capital, and water inrush can stimulate the change of the groundwater field at the same time. The process and mechanism of water inrush are similar to water inflow of working face, the hydrogeological parameters obtained by water inrush are more in line with the needs of the water disaster prevention and control in coal mine.

\section{The hydrogeological condition and water inrush situation in coal mine}

\section{Hydrogeological condition in coal mine}

A mine is located in the middle of Ningdong mining area of west margin of Ordos basin. The main mining coal seam is 2\#coal seam in Yan'an Formation of Jurassic, and its main water filling aquifer is the lower section of Zhiluo Formation aquifer that distributed throughout the coal field widely. The thickness of this aquifer is $60.21 \sim 317.70 \mathrm{~m}$, and the average thickness is $100.70 \mathrm{~m}$ (Fig.1). The lithology is gray-green, gray-brown, blue-gray medium and coarse sandstone with purple patches, 
mixed with a small amount of siltstone and mudstone, and the bottom contains gravel. According to the results of the pumping test, the permeability coefficient is $0.013 \sim 0.956 \mathrm{~m} / \mathrm{d}$, the unit water inflow is $0.036 \sim 0.218 \mathrm{~L} / \mathrm{s} \bullet \mathrm{m}$, and the water abundance is weak to medium.

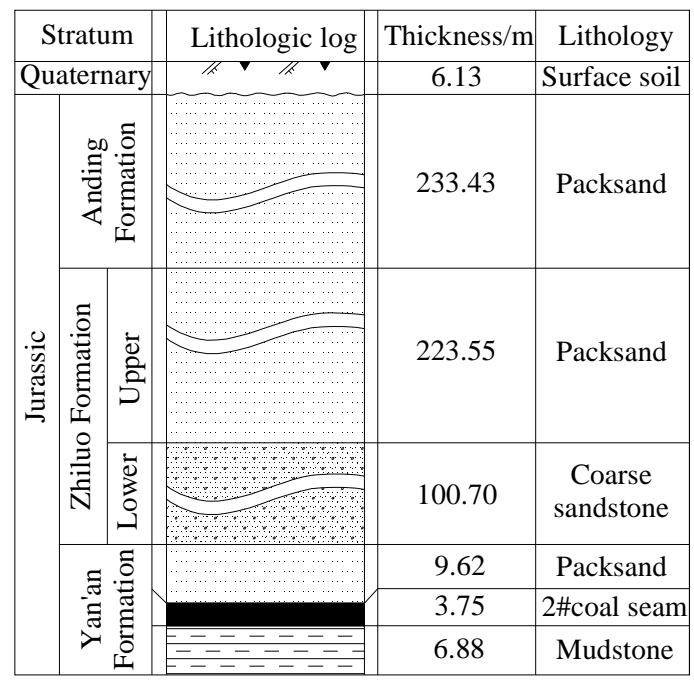

Fig.1 Integrated column schematic diagram of coal mine

\section{Process of water inrush}

The hydrogeological condition of lower section of Zhiluo Formation aquifer on the 2\#coal seam is complicated. The water disaster was happened during the tunnel of 2\#coal seam excavating, and the peak water inflow was about $400 \mathrm{~m}^{3} / \mathrm{h}$. Through the process of water inrush and hydrogeological condition analysis, the water inrush source was the lower section of Zhiluo Formation aquifer on the tunnel, and the channel is the crack of rock. After the occurrence of water inrush, the water level of long observation borehole in the lower section of Zhiluo Formation aquifer decreased, and the water level of ZL5 and ZL6 long-time observation borehole near the water inrush point decreased significantly (Fig.2).

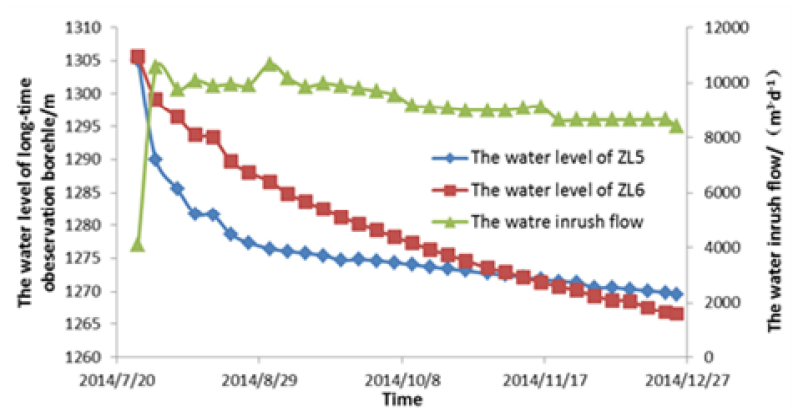

Fig. 2 The change curve of water inrush quantity and neighboring long-term observation hole

\section{The permeability coefficient inversion based on numerical simulation}

\section{Hydrogeological conceptual model}

The hydrogeological conceptual model is the basic model of the numerical simulation and physical simulation through the actual generalization of boundary property, internal structure, permeability, hydraulic characteristics and recharge, runoff, discharge conditions for aquifer. The source of water inrush is the lower section of Zhiluo Formation aquifer that can be regard as heterogeneous anisotropy aquifer. The research area is the first mining area, the southeast boundary of the area is Yujialiang fault, and the northeast boundary is F10 fault. The lower section of Zhiluo Formation join with aquiclude in the Yujialiang and F10 fault, these faults are water resistance on the horizontal direction and non-water conductive on the vertical direction. These two boundaries can be generalized as the second kind of water boundary. Due to the $2 \#$ coal seam is not mining, the lower section of Zhiluo Formation aquifer is not disturbed, and the scope of depression cone is limited, the northeast and southeast boundary can be generalized as the first kind of water boundary (Fig.3). 


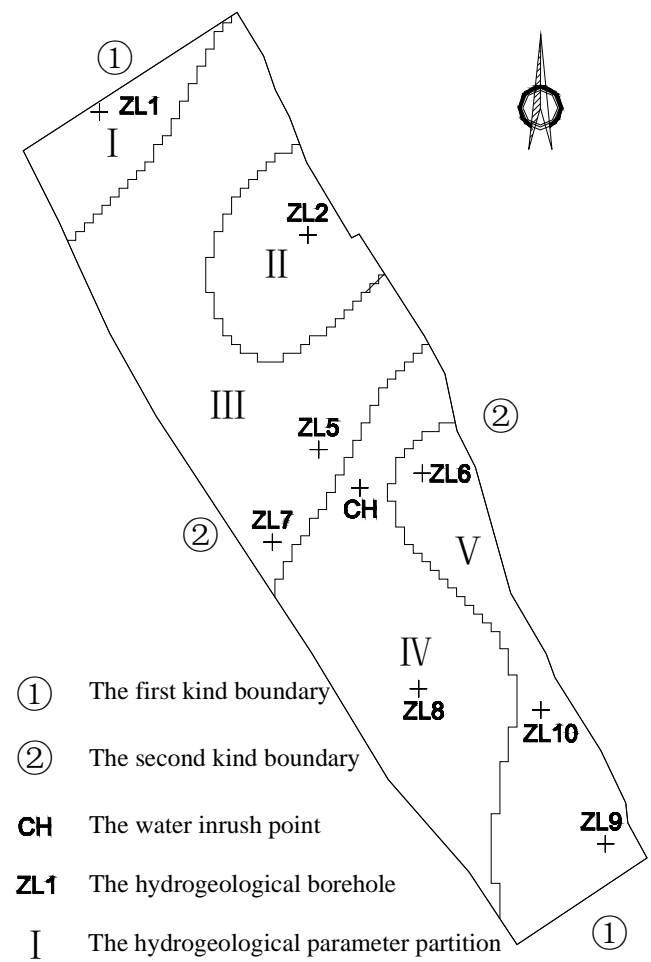

Fig.3 The hydrogeological conceptual model in the study area

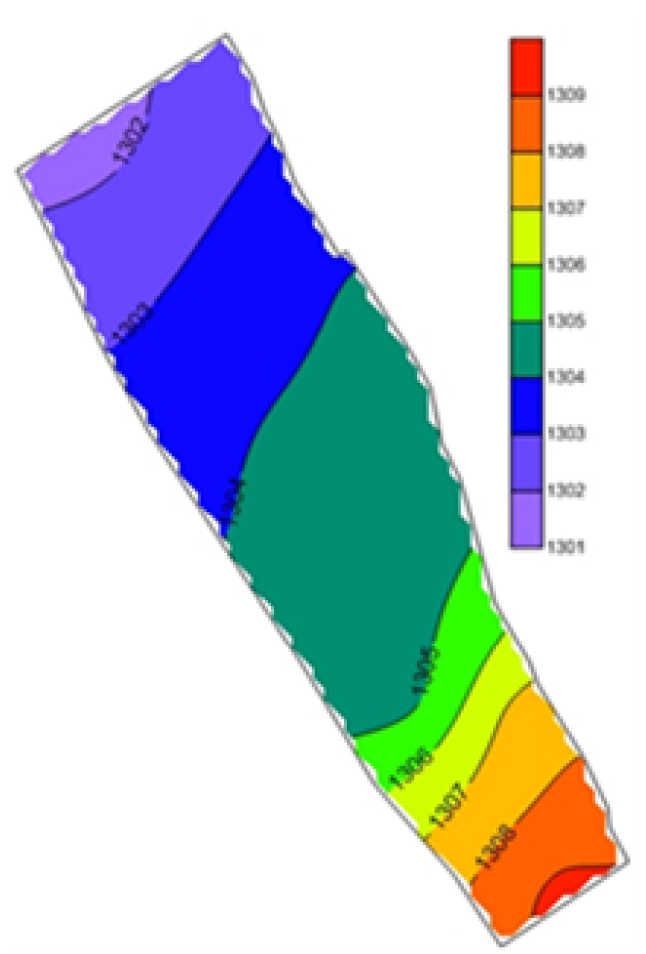

Fig.4 Initial groundwater flow field in the study area

There is a thick aquiclude with poor permeability on the lower section of Zhiluo Formation aquifer, the recharge of precipitation and surface water to the lower section of Zhiluo Formation aquifer can be ignored. Therefore, the main recharge of the research area is lateral recharge of groundwater, the water inrush and the lateral discharge of groundwater is the main discharge.

\section{Mathematical model}

After the analysis, the aquifer system of the lower section of Zhiluo Formation aquifer can be generalized as a single two-dimensional heterogeneous anisotropy confined aquifer system. The mathematical model is :

$$
\begin{aligned}
& \frac{\partial}{\partial x}\left(K \frac{\partial}{\partial x}\right)+\frac{\partial}{\partial y}\left(K \frac{\partial}{\partial y}\right)+W=\mu^{*} \frac{\partial}{\partial t} \ldots(x, y) \in \Omega \\
& h(x, y, t)=h_{0}(x, y) \ldots(x, y) \in \Omega \\
& \left.h(x, y, t)\right|_{\Gamma_{1}}=h(x, y) \ldots(x, y) \in \Gamma_{1} \\
& \left.K \frac{\partial h}{\partial n}\right|_{\Gamma 2}=q(x, y, t) \ldots(x, y) \in \Gamma_{2}
\end{aligned}
$$

Where: $K$ — permeability coefficient $/\left(\mathrm{m}^{\circ} \mathrm{d}^{-1}\right) ; h —$ water level $/ \mathrm{m} ; W$ - source term $/\left(\mathrm{m}^{3} / \bullet \mathrm{h}^{-1}\right) ; t \_$time $/ \mathrm{h} ; \mu^{*}$ — the water storage rate $/ 1 \bullet \mathrm{m}^{-1} ; \Omega \_$the research area; $\Gamma_{1}$-- the first kind boundary; $\Gamma_{2} \longrightarrow$ the second kind boundary; $h_{0}(\mathrm{x}, \mathrm{y}) \longrightarrow$ initial water level; $h(\mathrm{x}, \mathrm{y})-$ water level function on the first type of boundary; $n-$ the outer normal direction of the second kind boundary; $q(\mathrm{x}, \mathrm{y}, \mathrm{t})$ - the known flow function of the second kind boundary.

\section{Parameters partition}

The groundwater flow numerical simulation software-Visual Modflow was used to calculating in the numerical model. The hydrogeological parameters of the research area came from hydrogeological exploration in the first mining area. According to the thickness, depth, lithology, groundwater flow field of the lower section of Zhiluo Formation aquifer, and combined with the pumping test data of hydrogeological borehole, the research area was divided into five partitions based on the hydrogeological parameters, as shown in Fig.3. 


\section{Mesh generation}

The research area was divided into 120 rows and 80 columns according to $100 \mathrm{~m} \times 100 \mathrm{~m}$ grid, the total grids is 9600 which 2885 were effective. The simulation time was $154 \mathrm{~d}$ from water inrush on July 26 to December 24.

\section{Initial flow field}

Before the water inrush, there is no water flow discharge, the groundwater flow field can be determined based on the hydrogeological exploration data. The groundwater flow in lower section of Zhiluo Formation aquifer was from southeast to northwest roughly (Fig.4).

\section{Analysis of model simulation results}

The fitting curve of observed data and calculated data of ZL5 and ZL6 long-time observation borehole were shown in Fig.5 and Fig.6. The numerical calculation results were closed to actual water levels in each period from the figures, and the errors were small.

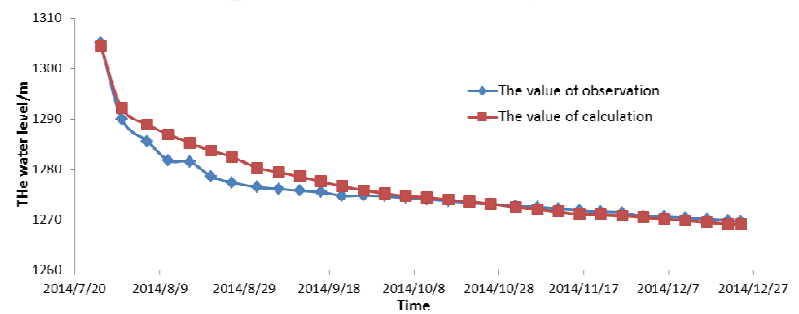

Fig.5 The water level fitting curve of long-term observation hole ZL5

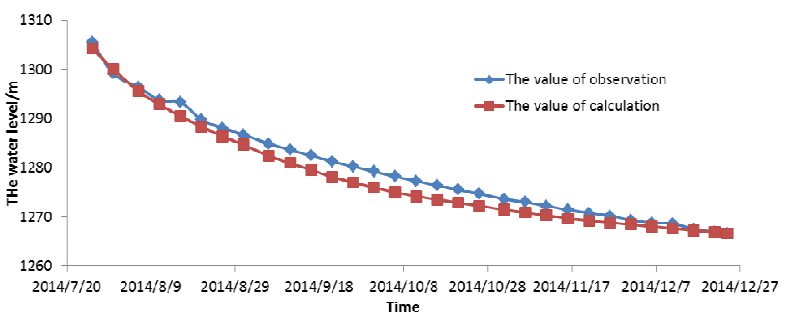

Fig.6 The water level fitting curve of long-term observation hole ZL6

The water level fitting precision of ZL5 and ZL6 long-time observation borehole is shown in Fig.7. The error between observed data and calculated data is small and within the $95 \%$ confidence interval. The numerical simulation model is in line with the actual hydrogeological condition.

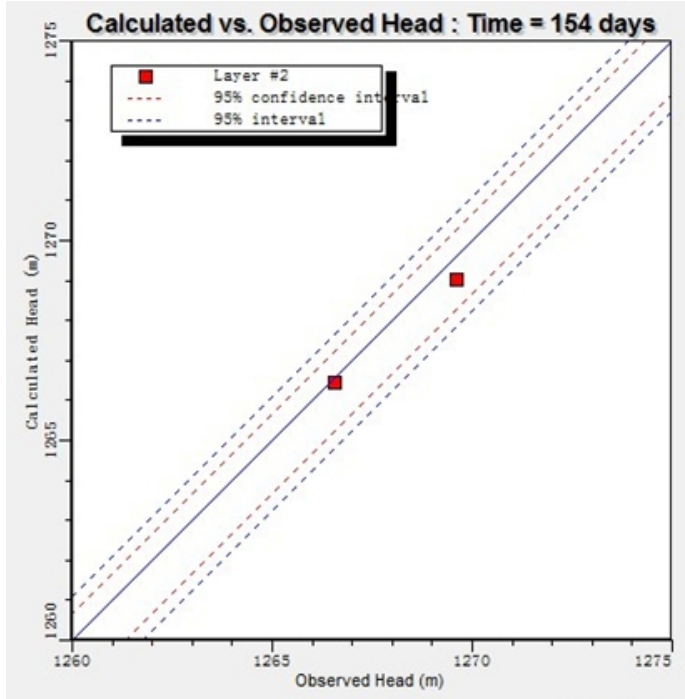

Fig.7 The water level fitting precision graph of long-term observation ZL5 and ZL6

According to the revised numerical model, the hydrogeological parameters of each zone in the research area were obtained (Table 1).

\section{Table1 Hydrogeological parameters of each subarea}

\begin{tabular}{ccc}
\hline Parameter partition & Permeability coefficient $/ \mathrm{md}^{-1}$ & Water storage rate $/ 1 \cdot \mathrm{m}^{-1}$ \\
\hline I & 1.5 & $2 \times 10^{-6}$ \\
II & 4.1 & $7 \times 10^{-5}$ \\
III & 3.0 & $6 \times 10^{-6}$ \\
IV & 1.1 & $5 \times 10^{-6}$ \\
V & 0.5 & $4 \times 10^{-6}$ \\
\hline
\end{tabular}




\section{Discussions}

In the past, the dewatering test of lower section of Zhiluo Formation aquifer was carried out, and the hydrogeological condition was identified and the hydrogeological parameters were obtained (Table 2).

Table2 Permeability coefficient of previous hydrogeological work $(\mathrm{m} / \mathrm{d})$

\begin{tabular}{ccccccc}
\hline Source of parameter & I & II & III & IV & V & Average vlue \\
\hline Dewatering test & $/$ & $/$ & 4.958 & $/$ & $/$ & 4.958 \\
Water inrush & 1.5 & 4.1 & 3.0 & 1.1 & 0.5 & 2.04 \\
\hline
\end{tabular}

According to the dewatering test data, the hydrogeological parameters from dewatering test and water inrush are closed to the actual situation. The permeability coefficient was determined for each hydrogeological parameter partition. The result can be the basic information for water disaster prevention and control in coal mine, and it also has a high reliability and practicability.

\section{Conclusions}

(1)According to the water inrush happened in 2\#coal seam tunnel, the hydrogeological model was established based on the Visual Modflow software, the numerical calculation results were closed to actual water levels and the permeability coefficient from the model were closed to the results of dewatering test. The numerical model has a high reliability.

(2)The hydrogeological condition of 2\#coal seam is complicated, and it is mainly threatened by the lower section of Zhiluo Formation aquifer. According to the inversion of the permeability coefficient of the aquifer, the permeability coefficient is $0.5 \sim 4.1 \mathrm{~m}^{\bullet} \mathrm{d}^{-1}$, and the water storage rate is $2 \times$ $10^{-6} \sim 7 \times 10^{-5}$. The heterogeneity of the aquifer permeability was indicted.

(3)The reversed permeability coefficient based on the water inrush data is close to dewatering test, which conforms to the hydrogeology condition of the lower section of Zhiluo formation aquifer, and it can be used as the basis for water prevention and control in coal mine.

\section{Acknowledgements}

This work was financially supported by the National key research and development plan (2016YFC0501104, 2016YFC0600708).

\section{References}

[1] D C WANG, R Q ZHANG and Y H SHI, et al. General Hydrogeology (The Geological Publishing House, China 2005).

[2] Y Q XUE, J C WU. Groundwater Dynamics 3rded.(The Geological Publishing House, China 2010). 